\title{
A Rare Case of Retroperitoneal Liposarcoma and Castleman's Disease
}

\section{ABSTRACT}

Retroperitoneal liposarcoma is a malignant mesenchymal tumour. The incidence is rare and early diagnosis is difficult as they present with non specific symptoms. A 55-year-old male patient, presented to General Surgery Department with complaints of abdominal swelling since two months and discomfort for three to four months. Abdominal examination revealed a mass, soft to firm in consistency with restricted mobility, was palpated in right quadrants. A clinical diagnosis of soft tissue tumour was made. Abdominopelvic computed tomography (CT) scan was performed showing a well-defined circumscribed, low-attenuated mass of retroperitoneal origin crossing midline and significantly displacing the intestinal loops, right grade 2 hydronephrosis and was compressing on inferior vena cava posteriorly. The patient underwent a resection surgery of the lesion and the histopathological examination showed a well-differentiated liposarcoma and Castleman's disease. These tumours are generally resistant to radiotherapy and/or chemotherapy. Thus the major prognostic factor related to survival is resection with tumour-free margins that includes the tumour as well as adjacent structures showing infiltration.

Keywords: Lymphoproliferative, Mesenchymal tumour, Sarcoma, Soft tissue

\section{CASE REPORT}

A 55-year-old male patient presented to the General Surgery Department with the complaint of abdominal swelling since two months. Patient also complaint of discomfort for three to four months duration followed by the swelling. With recent onset of dull, intermittent and non radiating pain in the right upper abdomen since two weeks. The pain had no specific aggravating or relieving factors. There was associated nausea and vomiting. Vomiting was non projectile in nature, containing partially digested food particles, non foul smelling, non bilious and was not blood stained. During the same two weeks, patient also complained of decreased appetite and generalised fatigue. No similar episodes or complaints in the past were noted.

On physical examination, the vitals recorded were pulse rate $82 / \mathrm{min}$, blood pressure 130/80 $\mathrm{mmHg}$ and respiratory rate $21 / \mathrm{min}$. Per abdomen examination showed fullness along right abdominal quadrants and a mass of $15 \times 12 \mathrm{~cm}$ with no clear margins was palpated in the right quadrants extending to midline at the level of umbilicus. The mass was soft to firm in consistency, did not move with respiration or fall forwards. Mass was dull on percussion with no evidence of free fluid. Laboratory investigations showed low Haemoglobin-8.2 g/dL, total leucocyte count of 5000 cells/cumm and other parameters (renal and liver function tests) were within normal limits. On performing Ultrasonography (USG) abdomen, an ill-defined heterogeneous mass occupying right hypochondrium, right lumbar and right iliac fossa was reported [Table/Fig-1].

With origin being uncertain, thoracic-abdominopelvic contrast enhanced computed tomography (CECT) performed showed a large well-circumscribed low attenuated mass lesion with epicentre in right anterior pararenal space measuring $26 \times 22.9 \times 14.5 \mathrm{~cm}$ crossing the midline to left with compression over right kidney and renal pelvis posteriorly. Mixed areas with minimal calcification and areas of necrosis were noted. Renal pelvis stretched and the ureter displaced anteromedially with resultant Grade 2 hydronephrosis [1]. The mass was visualised abutting the inferior surface of liver, gallbladder and inferior vena cava. The small bowel was displaced to opposite side. The CECT imaging showed no local nodal or distant metastases [Table/Fig-2]. No differential diagnosis was made in view of its retroperitoneal origin and clinical correlation.

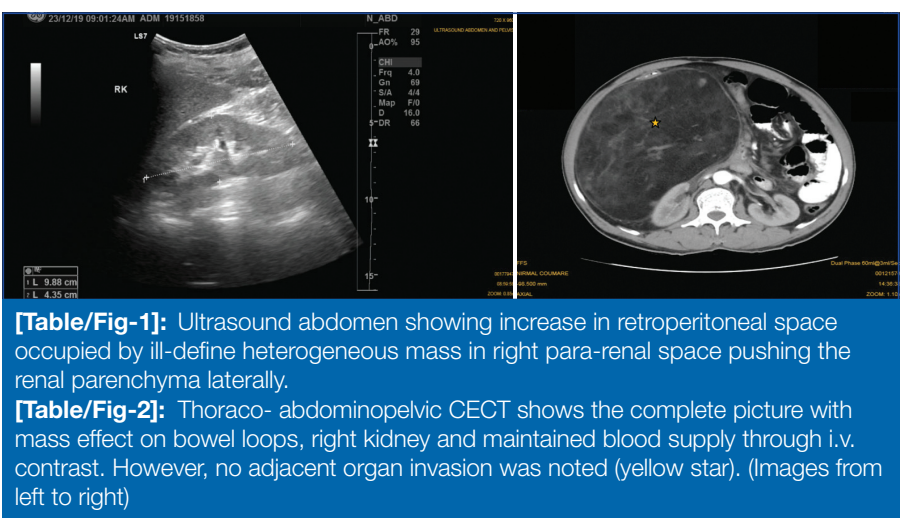

Preanaesthetic evaluation showed no contraindication except for low haemoglobin which was optimised using two units of Packed Red Blood Cells (PRBC). With adequate cardio pulmonary status, reserved additional PRBC, patient underwent laparotomy three weeks from time of presentation with the aim of en-bloc resection.

In view of stretched right ureter, Double $\mathrm{J}$ (DJ) stenting was done. This was followed by transverse incision two to three $\mathrm{cms}$ below the umbilicus extending between both anterior superior iliac spine. A large lipomatous tumour measuring $29 \times 28 \times 17 \mathrm{~cm}$ and weighing $4.8 \mathrm{~kg}$ was extracted from the retroperitoneal space with meticulous dissection without solid organ or visceral organ involvement [Table/ Fig-3,4]. Although the large mass displaced bowel, it was returned to normal position. No secondary lesions in the liver or abdominal nodes was identified.

The histopathological examination showed circumscribed tumour composed of adipocytes separated by fibrous septa. Individual adipocytes showing nuclear vacuolation with occasional lipoblasts corresponding to low-grade, well-differentiated liposarcoma and according to American Joint Committee on Cancer eighth edition [2] for soft tissue sarcomas arising from retroperitoneum, the staging was T4NOMO with Grade 1 differentiation. Also, a section adjacent to the lesion showed dense lymphoid aggregates with germinal centres separated by wide band of collagens and plasma cell resembling Castleman's disease. There was no evidence of lymphoma [Table/Fig-5a,b]. However, the resected margins were free of tumour infiltration. No specific treatment for castleman 
disease was given due to its incidental diagnosis and treatment is primarily surgical for unicentric disease.
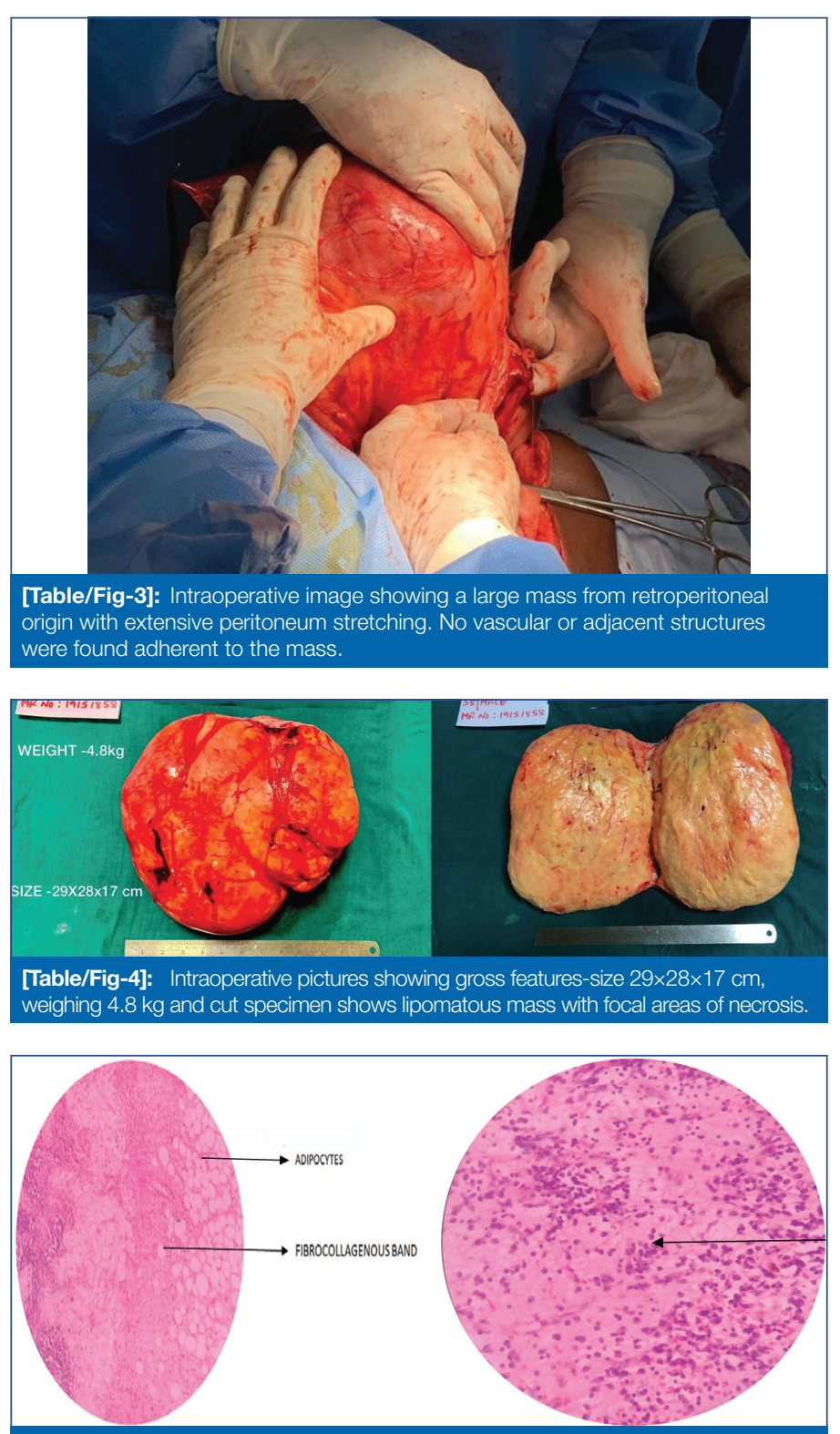

[Table/Fig-5a]: A 40x showing adipocytes and fibrocollagenous band under haematoxylin and eosin (H\&E) staining.

[Table/Fig-5b]: Further examination under 100X (H\&E) staining showed lymphoid aggregates and germinal centres separated by bands of collagen and plasma cells (black arrow). (Images from left to right)

The patient recovered without complications and was discharged on twelfth postoperative day. Patient was followed-up at regular intervals for the last two years and was uneventful for any operative complications or recurrence. This was confirmed using USG abdomen and pelvis at six months and one year with no significant changes [Table/Fig-6a,b]. Currently, the patient did not receive any chemo/radiotherapy and is on yearly follow-up.
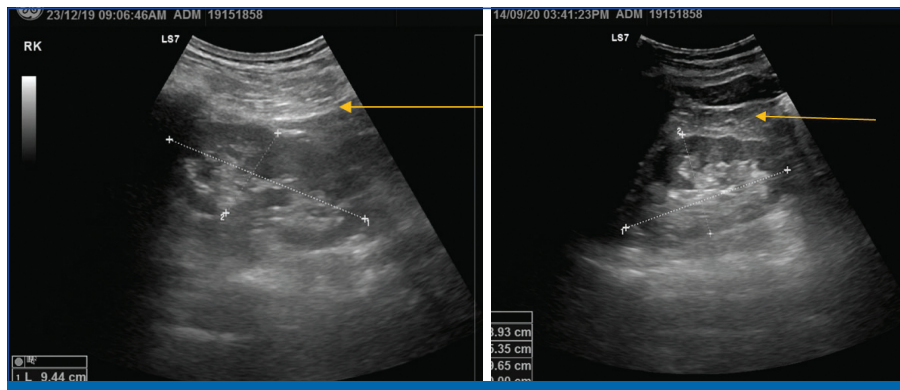

[Table/Fig-6a]: Postoperative follow-up with Ultrasonography (USG) abdomen and pelvis at first sixth month showed no abnormality or recurrence adjacent to and pelvis at
right kidney.

[Table/Fig-6b]: Second follow-up with USG at one year also showed no abnormality or recurrence adjacent to right kidney. (Images from left to right)

\section{DISCUSSION}

A wide spectrum of rare tumours arises from retroperitoneum that includes both benign and malignant tumours. Symptoms and imaging are considered non specific and histological diagnosis is confirmatory. Soft tissue sarcomas are relatively rare, among which retroperitoneal sarcomas constitute $10-15 \%$ of all soft tissue sarcomas. They are mesenchymal tumours and malignant tendency is less than $1 \%$ in adults. The reversible posterior leukoencephalopathy syndrome (RPLS) was first described by Giovanni Battista Morgagni in 1761 while performing autopsy of a 60-year-old woman. They are uncommon malignant tumours and a low incidence of approximately 4-5 per 100,000 individuals. It can affect both genders and age groups of 4060 years [3].

The prognosis depends highly on tumour margins and grading than tumour size. According to World Health Organisation (WHO) classification, they were categorised as well differentiated, dedifferentiated, myxoid, pleomorphic and mixed type. The well differentiated liposarcoma are identified as five histological variants [4]. The clinical manifestations vary from vague abdominal pain or mass to mass effect based on tumour growth. The treatment of choice is complete surgical excision and it is reported that no survival benefits of adding adjuvant chemotherapy or radiotherapy [5-7].

Another rare retroperitoneal fibrotic disease namely the Castleman's disease also has been reported with similar incidence in age and tumour location [8]. This condition presents as lymphoproliferative disease of lymph nodes. The pathogenesis is unclear but possible immunodeficiency and human herpes virus( $\mathrm{HHV}$ ) has been studied. Although commonly involves the mediastinal nodes, retroperitoneum has also been reported [9]. This condition also involves the same age group 40-60 years and equal gender distribution. The reported case here, showed similar age and the presentation as vague constitutional symptoms with abdominal mass [10]. However, the differentiating features further depends on centricity where unicentric castleman tends to be asymptomatic and multicentric castleman disease can present with constitutional symptoms and carries poor prognosis. This disease is not malignant condition however it increases risk of other tumours especially B-cell lymphomas and follicular dendritic cell sarcomas [11].

Retroperitoneal liposarcomas are diagnosed based on non specific or constitutional symptoms due to the mass or incidentally on imaging. The investigation of choice is CECT allowing an adequate staging and preoperative evaluation. Pelvic magnetic resonance is used for ruling out muscular or vascular invasion, satellite lesions or recurrences [12]. The possible differential diagnosis mainly includes tumours arising from retroperitoneal visceral (renal or adrenal, pancreas) structure, a lymphoma or metastatic lesion. There are instances where collision tumours, atypical breast liposarcoma and synovial liposarcoma have been reported [13-15]. While the diagnosis of castleman disease is mostly a postoperative or histopathological diagnosis [8].

Based on the prognostic factor established by $\mathrm{WHO}$, the optimal treatment for retroperitoneal liposarcoma is en-bloc resection with or without adjacent viscera $[4,5,16]$. Although tumours are insensitive to chemo/or radiotherapy, adjuvant radiotherapy for tumours $>5 \mathrm{~cm}$ with positive surgical margins has shown to reduce recurrence rate without increasing survival. Thus, complete resection with free margins is the most important prognostic factor. Extensive resection for tumours in contact with adjacent organs tends to increase overall morbidity $[7,12,17,18]$. The case reported here shows retroperitoneal liposarcomas could grow faster to large size and may not involve the adjacent structures. This case report shows a rarity in co-existence of well-differentiated tumour and a premalignant disease.

\section{CONCLUSION(S)}

Retroperitoneal tumours are rare and presents with non specific symptoms due to its location and are often diagnosed by imaging. 
However, this requires confirmation by histopathology and the prognosis depends on en-mass surgical resection which was achieved in present case report.

\section{REFERENCES}

[1] Laurence S Baskin; Overview of fetal hydronephrosis. Version Version 29.0. UpToDate. Retrieved 2017-04-25. Last updated Apr 20, 2017 https://www. uptodate.com/contents/fetal-hydronephrosis-etiology-and-prenatal-management.

[2] Tanaka K, Ozaki T. New TNM classification (AJCC eighth edition) of bone and soft tissue sarcomas: JCOG Bone and Soft Tissue Tumour Study Group. Jpn J Clin Oncol. 2019;49(2):103-07.

[3] Matthyssens LE, Creytens D, Ceelen WP. Retroperitoneal liposarcoma: Current insights in diagnosis and treatment. Front Surg [Internet]. 2015 Feb 10 [cited 2021 Aug 12];2. Available from: http://journal.frontiersin.org/Article/10.3389/ fsurg.2015.00004/abstract.

[4] Fletcher CDM, editor. Pathology and genetics of tumours of soft tissue and bone. Lyon: IARC Press; 2002. 427. Pp. (World Health Organization Classification of Tumours).

[5] Caizzone A, Saladino E, Fleres F, Paviglianiti C, laropoli F, Mazzeo C, et al. Giant retroperitoneal liposarcoma: Case report and review of the literature. Int J Surg Case Rep. 2015;9:23-26. Doi: 10.1016/j.jjscr.2015.02.019.

[6] Wanchick K, Lucha P. Dedifferentiated retroperitoneal liposarcoma presenting as lower gastrointestinal bleeding, a case report and review of the literature. Mil Med. 2009;174(3):328-30.

[7] Pisters PWT, O'Sullivan B. Retroperitoneal sarcomas: Combined modality treatment approaches: Curr Opin Oncol. 2002;14(4):400-05.

[8] Masaki Y, Kawabata H, Fujimoto S, Kawano M, Iwaki N, Kotani T, et al. Epidemiological analysis of multicentric and unicentric Castleman disease and TAFRO syndrome in Japan. J Clin Exp Hematop JCEH. 2019;59(4):175-78.
[9] Polizzotto MN, Uldrick TS, Wang V, Aleman K, Wyvill KM, Marshall V, et al. Human and viral interleukin-6 and other cytokines in Kaposi sarcoma herpesvirusassociated multicentric Castleman disease. Blood. 2013;122(26):4189-98.

[10] Yu L, Tu M, Cortes J, Xu-Monette ZY, Miranda RN, Zhang J, et al. Clinical and pathological characteristics of HIV- and HHV-8-negative Castleman disease. Blood. 2017;129(12):1658-68.

[11] Keller AR, Hochholzer L, Castleman B. Hyaline-vascular and plasma-cell types of giant lymph node hyperplasia of the mediastinum and other locations. Cancer. 1972;29(3):670-83.

[12] Soft tissue and visceral sarcomas: ESMO Clinical Practice Guidelines for diagnosis, treatment and follow-up. Ann Oncol. 2012;23:vii92-99.

[13] Buchner D, Hieggelke L, Löser H, Bruns CJ, Quaas A, Ghadimi MPH. First reported case of a collision tumour composed of pancreatic adenocarcinoma and retroperitoneal liposarcoma: A case report. BMC Cancer. 2018;18(1):1243.

[14] Briski LM, Jorns JM. Primary breast atypical lipomatous tumour/welldifferentiated liposarcoma and dedifferentiated liposarcoma. Arch Pathol Lab Med. 2018;142(2):268-74.

[15] Wong C, Harris A, Kennedy R, Houghton O, Carey P. A rare case of retroperitoneal synovial sarcoma. JRSM Open. 2018;9(4):205427041876043.

[16] Singer S, Antonescu CR, Riedel E, Brennan MF. Histologic Subtype and Margin of Resection Predict Pattern of Recurrence and Survival for Retroperitoneal Liposarcoma. Ann Surg. 2003;238(3):358-71.

[17] Zhang WD, Liu DR, Que RS, Zhou CB, Zhan CN, Zhao JG, et al. Management of retroperitoneal liposarcoma: A case report and review of the literature. Oncol Lett. 2015;10(1):405-09.

[18] Lewis JJ, Leung D, Woodruff JM, Brennan MF. Retroperitoneal soft-tissue sarcoma: analysis of 500 patients treated and followed at a single institution. Ann Surg. 1998;228(3):355-65.

\section{PARTICULARS OF CONTRIBUTORS:}

1. Postgraduate, Department of General Surgery, Mahatma Gandhi Medical College and Research Institute, Puducherry, India.

2. Senior Resident, Department of General Surgery, Mahatma Gandhi Medical College and Research Institute, Puducherry, India.

3. Professor, Department of General Surgery, Mahatma Gandhi Medical College and Research Institute, Puducherry, India.

4. Professor, Department of General Surgery, Mahatma Gandhi Medical College and Research Institute, Puducherry, India

\section{NAME, ADDRESS, E-MAIL ID OF THE CORRESPONDING AUTHOR} Haresh Kumar Gobu,

2/37, VIP Nagar, Vilankurichi Road, Coimbatore-641035, Tamil Nadu, India E-mail: hareshg06@gmail.com

\section{AUTHOR DECLARATION:}

- Financial or Other Competing Interests: None

- Was informed consent obtained from the subjects involved in the study? Yes

- For any images presented appropriate consent has been obtained from the subjects. Yes
PLAGIARISM CHECKING METHODS: Jain Het al.]

- Plagiarism X-checker: May 04, 2021

- Manual Googling: Oct 01, 2021

- iThenticate Software: Oct 23, 2021 (1\%)
ETYMOLOGY: Author Origin

Date of Submission: May 03, 2021 Date of Peer Review: Jul 29, 2021 Date of Acceptance: Oct 01, 2021 Date of Publishing: Jan 01, 2022 